The Egyptian Journal of Hospital Medicine (July 2020) Vol. 80, Page 696-703

\title{
Comparative Anatomical Studies on the Skull of Three Mammals in Egypt Having Different Diets
}

Fatma M.A. Taki-El-Deen ${ }^{1,2}$

1-Department of Biological and Geological Sciences, Faculty of Education, Ain Shams University, Egypt.

2- Department of Biology, College of Science, Qassim University, Buraidah, Al-Qassim, Kingdom of Saudi Arabia. ABSTRACT

Aim of the work: the present work was designed to study the anatomical structure of the skull of three mammalian animals live in Egypt. These animals were Guinea Pig (Cavia porcellus), Hedgehog (Hemiechinus auritus) and Least Weasel (Mustela nivalis vulgaris). It is worth mentioning that these animals are having different diets since, Cavia porcellus is herbivorous, Hemiechinus auritus is insectivorousand Mustela nivalis vulgaris is carnivorous.

Materials and methods: heads of the three animals (Cavia porcellus, Hemiechinus auritus and Mustela nivalis vulgaris) were used to study the anatomical structure of the skull and mandible in each animal.

Results: after preparation of each skull of the animals under investigation, it was photographed and described according to the different views; dorsal, ventral, lateral and occipital in addition to the lower jaw. It is worth mentioned that these animals had different diets. So, some differences were observed in the facial and cranial regions of the skulls in the three animals. The parietals and frontals in the skull of Cavia porcellus were slightly convex and the skull had a broad zygomatic arch. The skull of Hemiechinus auritus had a long facial region and the tympanic bulla was not well developed. The skull of Mustela nivalis had short facial region and long cranial region which had an obvious prominent sagittal crest. The lower jaw had two mandibles which were connected firmly by bony mandibular symphysis. Also, heterodont teeth were arranged on the upper and lower jaws.

Keywords: anatomy, skull, Guinea Pig, Hedgehog, Least Weasel, lower jaw

\section{INTRODUCTION}

The mammalian's skull encloses and protects the brain and sense organs. Also, it gives the special shape of the head ${ }^{(1)}$. The skull consists of many bones that are separated from each other by sutures. These bones are grouped in two main regions which are the facial and cranial $^{(2)}$. The occipital bones represent the caudal part of the skull ${ }^{(3)}$. There are two occipital condyles enclose the foramen magnum ${ }^{(4)}$. The dorsal surface of the cranial region comprisesthe parietal and frontal bones ${ }^{(5)}$. There is a nuchal crest at the line that connects the posterior border of the parietal and squamosal with the superior border of occipital bones ${ }^{(6)}$. Also, there is a prominent sagittal crest in the dorsal surface of the skull. The parietal bones are separated by the sagittal crest ${ }^{(7)}$.

In the bear's skull, the sagittal crest is not eminent (8). The squamosal is placed on the lateral side of the cranium. It articulates dorsally with the parietal bone. Squamosal bone participates in the formation of zygomatic arch by giving a zygomatic process of squamosal. The cheek region of the animal's head is supported internally by the zygomatic arch ${ }^{(9)}$.

The tympanic bullae are located on each side of the basioccipital bone. The pterygoids form the superior and lateral walls of the naso-pharyngeal passage. The vomer articulates caudally with the presphenoid. It forms the basal part of the nasal septum ${ }^{(9)}$. The orbital cavity contains the eye ball during the life of the animal. It may be bordered superiorly by supraorbital process or not. Also, the supra-orbital foramen is absent in some mammals ${ }^{(\mathbf{1 0})}$.

The nasal cavities are covered by the nasal bones. These cavities have scroll-shaped turbinal bones ${ }^{(11)}$. Both upper and lower jaws bear heterodont teeth. The lower jaw is formed of two mandibles which articulated anteriorly by bony mandibular symphysis ${ }^{(\mathbf{1 2})}$.

\section{MATERIALS AND METHODS}

The heads of the three animals (Cavia porcellus, Hemiechinus auritus and Mustela nivalis vulgaris) were used to study the anatomical structure of the skull and mandible in each animal. Preparation of the skulls was done by using boiling water to get rid of thesoft tissues. Then they were placed in 3\% hydrogen peroxide for two weeks. After that, the skulls were left in sunlight to bleach (13).

\section{RESULTS}

\section{Dorsal View of the Guinea Pig's skull:}

The skull of Guinea Pig is composed of the facial region and the cranial region. The cranial bones are: occipital, parietal, squamosal, sphenoid and ethmoid.

The facial bones are: frontal, nasal, lacrimal, zygomatic, vomer, palatine, maxilla and premaxilla. The

This article is an open access article distributed under the terms and conditions of the Creative Commons Attribution (CC BY-SA) license (http://creativecommons.org/licenses/by/4.0/) 
mid- dorsal suture which located between the parietal, frontal and nasal bones is slightly straight. The skull lacks the sagittal crest. But, the nuchal crest is obvious and it marks the boundary between the parietal and occipital bones (Fig. 1).

\section{Ventral view of Guinea Pig's skull:}

The rostral part of the skull is formed from the hard palate. The caudal part is formed from occipital bones which form the two occipital condyles (enclose the foramen magnum). The occipital bones are represented by the supraoccipital, basioccipital and two exoccipital. Two tympanic bullae which have a flask shaped are located on each side of the basioccipital bone. Each tympanic bulla has an external auditory opening which is called the external auditory meatus. The basioccipital bone articulates anteriorly with the basisphenoid bone which articulates rostrally with the presphenoid bone. The vomer bone is located in the midventral area and articulates posteriorly with the presphenoid. The teeth are heterodont and the incisors are located on the premaxilla. The diastema separates incisor from the premolar (Fig. 2).

\section{Lateral view of Guinea Pig's skull:}

The lateral view is characterized by the orbital cavity which bordered superiorly by the supraorbital process. The zygomatic arch components are:process of maxilla,jugal and process of squamosal. The jugal bone is a slightly broad bone. The squamosal bone is located on each side of the cranium. The tympanic bulla is well observed in this view. The lacrimal bone is a perforated bone and is placed in the anterior part of the orbit. There is an obvious distance between the incisor and first premolar which is called diastema (Fig. 3).

\section{Occipital view of Guinea Pig's skull:}

The occipital bones are forming the occipital ring which encloses the foramen magnum which has two occipital condyles. The occipital bones are: supraoccipital, basioccipital and two exoccipitals. The supraoccipital process joins the parietal bones at a nuchal crest. The basioccipital is located ventrally between the two exoccipitals which extend the supraoccipital alongside of each occipital condyle is present superiorly (Fig. 4).

\section{Lower jaw of Guinea Pig's skull:}

The lower jaw consists of two rami, each one is formed from the dentary bone which extends to articulate with the upper jaw. There are three distinct processes associated with the mandible which are; articular process, coronoid and angular (Fig.5).

The dental formula is as follow: (I 1/1, C0/0, P1/1, M3/3) x2 = 20 teeth.

\section{Dorsal view of Hedgehog's skull:}

The skull of Guinea Pig has two regions which are the facial and cranial. The convexity of parietal bones is greater than in Guinea Pig's skull. The frontal bones mainly form the roof of the skull. They articulated anteriorly with the nasal bones. The nasal bones cover the nasal cavities which has turbinal bones. The zygomatic arch is a slender- shaped structure. It consists of zygomatic processes (maxilla and squamosal) and the jugal bone. The sagittal crest is slightly developed and the nuchal crest is well developed (Fig. 6).

\section{Ventral view of Hedgehog's skull:}

Ventrally, the basioccipital bone is bordered anteriorly by the basisphenoid bone and laterally by the tympanic bulla which is not well formed. The palatine bones enclose the vomer. The two pterygoids meet at the midventral line of the skull. The premaxilla bears three incisors. While, the maxilla bears one canine, three premolars and three molars. The jugal bone is a slender stout bone and connects the maxilla anteriorly with the squamosal posteriorly (Fig. 7).

\section{Lateral view of Hedgehog's skull:}

Laterally, the skull is extended from the posterior edge of the orbit and ventrolateral edges of parietal and frontal. The zygomatic arch is smoothly curved and comprises the process of maxilla, jugal and process of squamosal. The orbital cavity has a lacrimal bone in the anterior corner of it. There is no supraorbital process or supra orbital foramen. The nasal bones cover the nasal cavities which enclose the thin turbinal bones (Fig. 8).

\section{Occipital view of Hedgehog's skull:}

The occipital bones are represented by supraoccipital , basioccipital and two exoccipitals. They enclose the semicircular foramen magnum. The lateral borders of the foramen magnum are guarded by curved occipital condyle. The exoccipital bones are located on each side of the dccipital condyle. The supraoccipital lies above the foramen magnum. The nuchal crest lies above the supraoccipital bone (Fig. 9).

\section{Lower jaw of Hedgehog's skull:}

The lower jaw has two rami. Each ramus (mandible) consists of one extending dentary bone and three other processes. The processes are: the coronoid process which represents the superior one, the articular process articulates with upper mandible and the angular process (Fig. 10). The dental formula is as follow: (I 3/3, C1/1, P3/2, M3/3) x2 = 38 teeth. 


\section{https://ejhm.journals.ekb.eg/}

\section{Dorsal view of Least Weasel's skull:}

The facial region is short but, the cranial region is slightly elongated. The dorsal view is characterized by a mid-longitudinal prominent crest extends from the anterior tip to the posterior end. The sagittal crest rostrally decreases in its height and divides at thefrontal bone to takes V-shaped. The parietal bones form the most part of the cranium with a little participates of the squamosal and frontal bones. The parietal bones marginated posteriorly by the well developed nuchal crest which lined above the supraoccipital bone.Nasals cover the nasal cavities which contain the delicate turbinal bones. The premaxillae and maxillae are located in the anterior part of the facial region(Fig. 11).

\section{Ventral view of Least Weasel's skull:}

The cranium is elongated and has an oval tympanic bulla on each side of the basioccipital bone. The basioccipital is attached anteriorly with the basisphenoid which lies at the posterior margin of the presphenoid. The basisphenoid fuses anteriorly-laterally with the pterygoid bone. The hard palate is well developed. The premaxilla bears the incisors. The heterodont teeth are located in the premaxilla and maxilla. Three incisors are located on the premaxillabone. The canine, three molars and one molar lie on the maxilla bone (Fig. 12).

\section{Lateral view of Least Weasel's skull:}

The zygomatic arch is well seen in this view. It is curved dorso-laterally and forms lower boundary of the temporal fossae. The squamosal bone lies on each side of the cranium and articulates dorsally with the parietal bone. Also, it gives the zygomatic process of squamosal. The maxilla bone gives the zygomatic process of maxilla. The jugal is a stout narrow bone. The heterodont teeth are arranged in the upper jaw. They are well seen in the lateral view. Also, the three incisors present on the anterior margin of premaxilla bone. But, the canine, premolar and molars lie on the maxilla bone (Fig. 13).

\section{Occipital view of Least Weasel's skull:}

The ring of the occipital bones is represented by two exoccipital, one supraoccipital and one basioccipital. The posterior borders of the parietal and squamosal bones are attached with the superior border of the occipital bones through a well developed nuchal crest. There are two occipital condyles located on each side of the oval foramen magnum (Fig. 14).

\section{Lower jaw of Least Weasel's skull:}

The lower jaw has two mandibles (rami). Each ramus (mandible) is characterized by straight lower margin. It consists of elongated dentary bone in addition to three processes. The superior process is the coronoid process. The articular process is located at the posterior edge of the mandible. The angular process located under the articular one. The premaxilla bone bears three incisors. While, the maxilla bone bears one canine, three premolars and two molars (Fig. 15). The dental formula is as follow:

(I 3/3, C1/1, P3/3, M1/2) x2 = 34 teeth. 


\section{Explanation of figures}
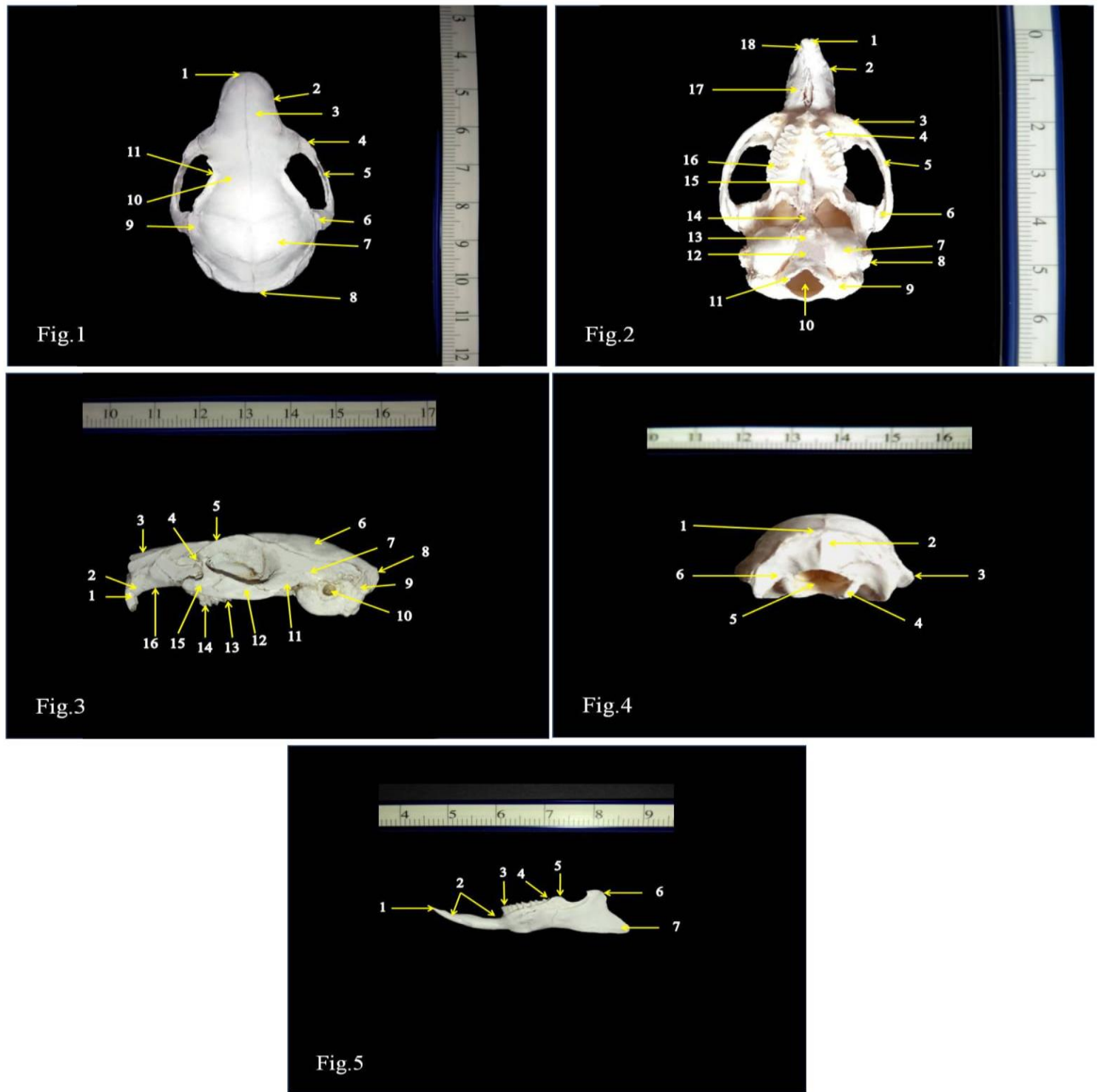

Fig. 1: photograph of the ventral view of Guinea Pig's skull showing: 1.Premaxilla; 2. Maxilla; 3. Nasal; 4. Zygomatic process of maxilla; 5. Jugal; 6. Zygomatic process of squamosal; 7. Parietal; 8. Nuchal crest; 9. Squamosal; 10. Frontal; 11. Supraorbital process

Fig. 2: photograph of the ventral view of Guinea Pig's skull showing: 1.Incisor; 2. Maxilla; 3. Zygomatic process of maxilla; 4. premolar; 5. Jugal; 6. Zygomatic process of squamosal; 7. Tympanic bulla; 8. External auditory meatus; 9. Exoccipital; 10. Foramen magnum; 11. Occipital condyle; 12. Basioccipital; 13. Basisphenoid; 14. Presphenoid; 15. Vomer; 16. Molar; 17. Diastema; 18. Premaxilla 
Fig. (3): photograph of the lateral view of Guinea Pig' skull showing: 1.incisor; 2. premaxilla; 3. nasal; 4. lacrimal; 5. frontal; 6. parietal; 7. squamosal; 8. supraoccipital; 9. exoccipital; 10. external auditory meatus; 11. zygomatic process of squamosal; 12. jugal; 13. molar; 14. premolar; 15. zygomatic process of maxilla; 16. maxilla.

Fig. 4: photograph of the occipital view of Guinea Pig's skull showing: 1.Nuchal crest; 2. Supraoccipital; 3. Mastoid process; 4. Occipital condyle; 5. Foramen magnum; 6. Exoccipital

Fig. 5: photograph of the mandible of Guinea Pig's skull showing: 1.Incisors; 2. Diastema; 3. Premolar; 4. Molar; 5. Articular; 6. Coronoid process; 7. Angular process
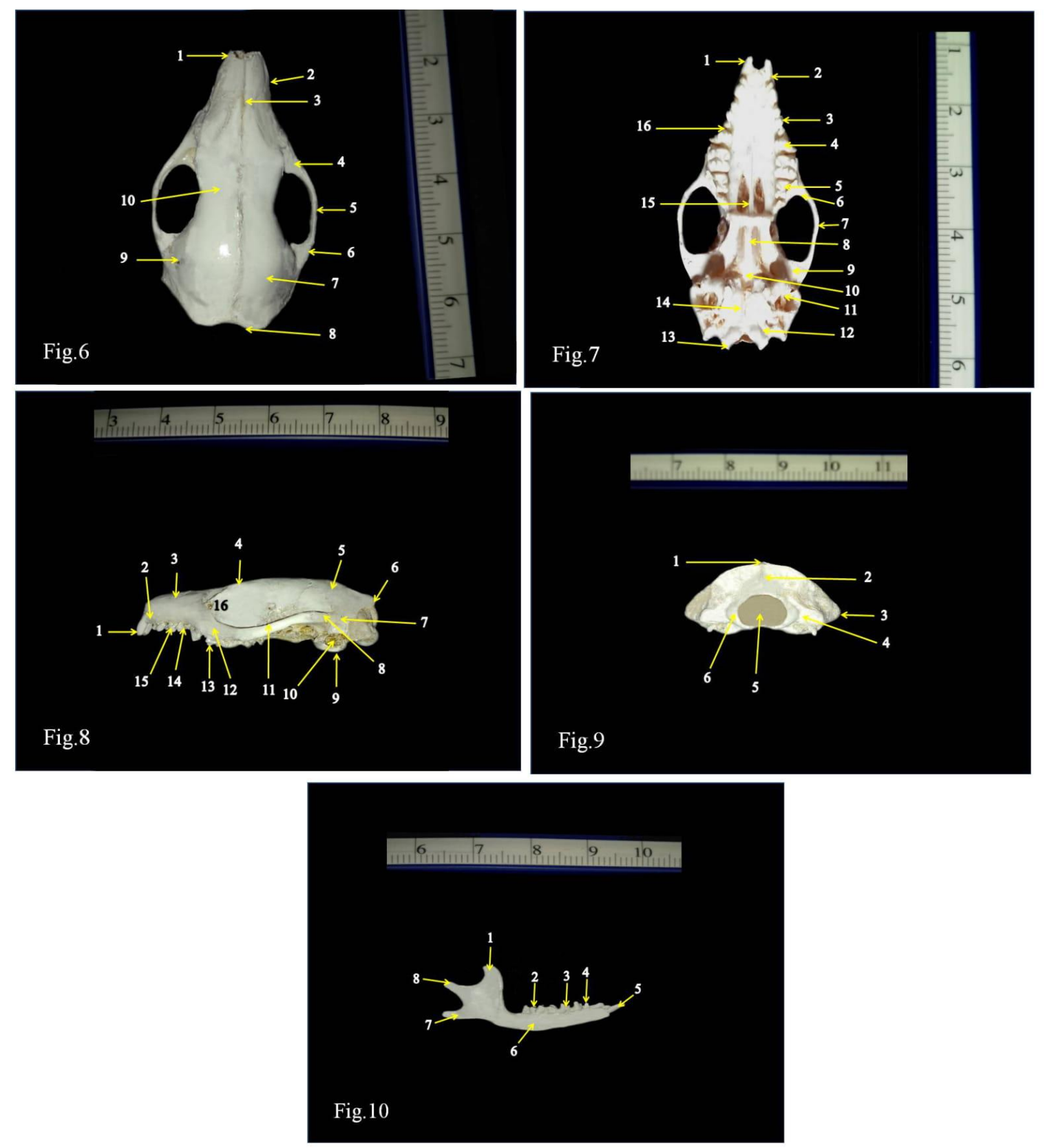

Fig. 6: photograph of the dorsal view of Hedgehog' skull showing: 1.Premaxilla; 2. Maxilla; 3. Nasal; 4.Zygomatic process of maxilla; 5. Jugal; 6. Zygomatic process of squamosal; 7.Pareital; 8. Nuchal crest; 9.Squamosal; 10. Frontal

Fig. 7: photograph of the ventral view of Hedgehog' skull showing: 1.Incisor; 2. Premaxilla; 3. Canine; 4. Premolar; 5. Molar; 6. Zygomatic process of maxilla; 7. Jugal; 8. Presphenoid; 9. Zygomatic process of squamosal; 10. Basispheniod; 11. Tympanic bulla; 12. Exoccipital; 13. Occipital condyle; 14. Basioccipital; 15. Vomer; 16.Maxilla 
Fig. 8: photograph of the lateral view of Hedgehog' skull showing: 1.Incisor; 2. Premaxilla; 3. nasal; 4. Frontal; 5. Parietal; 6. Nuchal crest; 7. Squamosal; 8. Zygomatic process of squamosal; 9. Tympanic bulla; 10. External auditory meatus; 11. Jugal; 12. Zygomatic process of maxilla; 13. Molar; 14. Premolar; 15. Canine; 16. Lacrimal Fig. 9: photograph of the occipital view of Hedgehog' skull showing: 1.Nuchal crest; 2. Supraoccipital; 3. Mastoid process; 4. Exoccipital condyle; 5. Foramen magnum; 6. Occipital condyle

Fig. 10: photograph of the mandible of Hedgehog' skull showing: 1.Coronoid process; 2. Molar ; 3. Premolar ; 4. Canine ; 5. Incisor ; 6. Dentary; 7. Angular process; 8. Articular process
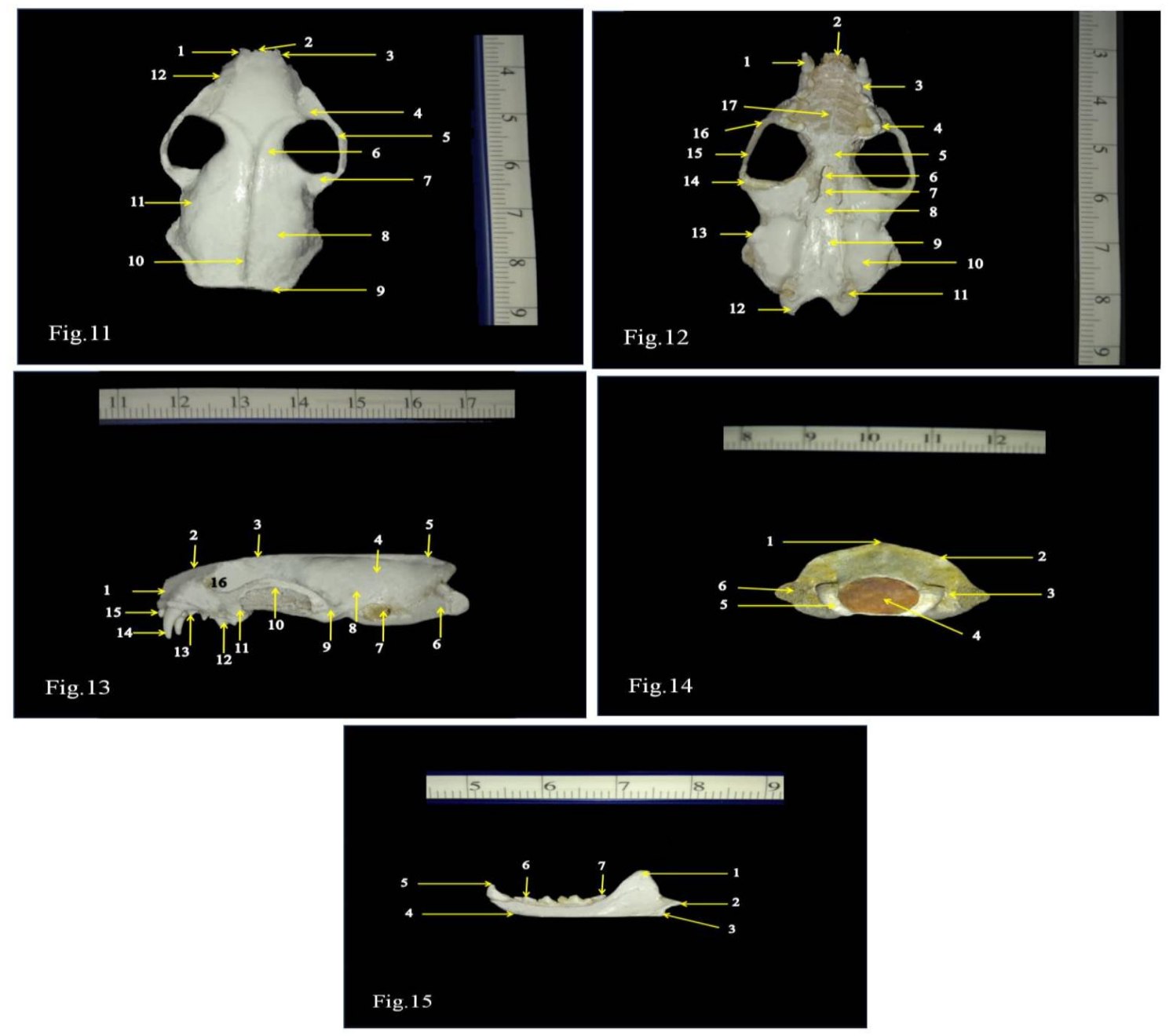

Fig. 11: photograph of the dorsal view of Least Weasel's skull showing: 1.Canine; 2. Incisor; 3. Premaxilla; 4.Zygomatic process of maxilla; 5. Jugal; 6. Frontal; 7. Zygomatic process of squamosal; 8. Parietal; 9. Nuchal crest; 10. Sagittal crest; 11.Squamosal; 12.Maxilla

Fig. 12: photograph of the ventral view of Least Weasel's skull showing: 1. Canine; 2. Incisor; 3. Premolar; 4. Molar; 5.Palatine; 6. Vomer; 7. Presphenoid; 8. Basispheniod; 9. Basioccipital; 10. Tympanic bulla; 11. Exoccipital; 12. Occipital condyle; 13. External auditory meatus; 14. Zygomatic process of squamosal; 15. Jugal; 16. Zygomatic process of maxilla; 17.Palatine 
https://ejhm.journals.ekb.eg/

Fig. 13: photograph of the lateral view of Least Weasel's skull showing: 1. Premaxilla; 2. Nasal; 3. Frontal; 4. Parietal; 5. Sagittal crest; 6.Exoccipital; 7. External auditory meatus; 8. Squamosal; 9. Zygomatic process of squamosal; tympanic bulla; 10. Jugal; 11. zygomatic process of maxilla; 12. Molar; 13. Premolar; 14 . Canine; 15. Incisor; 16. Lacrimal

Fig. 14: photograph of the occipital view of Least Weasel's skull showing: 1.Sagittal crest; 2 . Nuchal crest; 3 . Exoccipital ; 4. Foramen magnum; 5. Occipital condyle; 6. Mastoid process

Fig. 15: photograph of the mandible of Least Weasel's skull showing: 1.Coronoid process; 2. Articular process; 3. Angular process; 4. Dentary ; 5. Canine; 6. Premolar; 7. Molar

\section{DISCUSSION}

The skull is composed of two main regions; facial and cranial. The cranial bones are: occipital, parietal, squamosal, sphenoid and ethmoid. The facial bones are: frontal, nasal, lacrimal, zygomatic, vomer, palatine, maxilla and premaxilla. It is worth mention that the skull of Mustela nivalis is characterized by short facial region and long cranial region. In common opossum, the facial region is long and the cranial region is $\operatorname{long}^{(\mathbf{1 4})}$. The occipital ring forms the caudal part of the skull ${ }^{(\mathbf{9})}$. This ring is represented by occipital bones which are: two exoccipital, one supraoccipital and one basioccipital. The basioccipital is located ventrally between the two exoccipitals. The supraoccipital lies above the foramen magnum. The position of the occipital bones in the three skulls under investigation was found in the leopard cat (Felis bengalensis) described by Sarma et al. ${ }^{(3)}$. There are two occipital condyles which are placed on each side of the foramen magnum ${ }^{(\mathbf{1 5})}$.

The foramen magnum in the three skulls under this study takes oval shape. Similar observations were recorded in kangal dogs, badgers, martens and otters ${ }^{(\mathbf{1 6})}$. The sphenoid bone is palced anteriorly to the basioccipital. The ethmoid bone is located between the nasal cavity and the cranial cavity bone ${ }^{(6)}$.

In Cavia porcellus, the tympanic bullae have a flask shaped structure and located on each side of the basioccipital bone. Each tympanic bulla has an external auditory opening which is called the external auditory meatus. The tympanic bulla in Mustela nivalis is well developed $^{(\mathbf{1 7 )}}$. The tympanic bulla in Hemiechinus auritus is small in size and well developed ${ }^{(\mathbf{1 8})}$. While, the common opossum lacks the tympanic bulla but, it has a tympanic process of alisphenoid ${ }^{(14)}$.

The parietal bones are placed on the dorsal region of the cranium and form the most part of $\mathrm{it}^{(\mathbf{6})}$. The convexity of parietal bones in Cavia porcellus is greater than both Hemiechinus auritus and Mustela nivalis. The squamosal bone participates in the formation of the lateral wall of the cranium and articulates dorsally with parietal bone. Also, it gives the zygomatic process of squamosal. The posterior border of the parietal and squamosal bones is attached with the superior border of the occipital bones through a well developed nuchal crest $^{(\boldsymbol{6})}$. The skull of Mustela nivalis is characterized by a prominent sagittal crest $^{(\mathbf{1 9})}$. The sagittal crest in Mustela nivalis is more prominent than in Hemiechinus auritus. This crest decreases in its height and divides to take V-shaped at the position of frontal bones ${ }^{(20)}$. The skull of Cavia porcellus lacks the sagittal crest.The orbital cavity is bordered superiorly by the supra-orbital process. The supra - orbital foramen is absent in the three skulls under investigation $^{(\mathbf{2 1})}$.

In Cavia porcellus, the orbital cavity is bordered superiorly by the supra-orbital process. It is absent in Hemiechinus auritus and Mustela nivalis. In carinvores, there is an orbital ligament located in the dorsal region of the orbital cavity ${ }^{(22)}$. The frontal bones are convex in Cavia porcellus ${ }^{(\mathbf{2 3})}$. The frontal bones are paired and articulate anteriorly with the nasal bones ${ }^{(\mathbf{1 2})}$. Nasal bones cover the nasal cavities which contain delicate scrollshaped structures which are called the turbinal bones. These turbinal bones arise from the lateral walls of the nasal cavities ${ }^{(\mathbf{1 1})}$. The lacrimal bone is a perforated bone and is located in the anterior corner of the orbitl cavity $^{\left({ }^{(6)}\right.}$. The zygomatic arch has the zygomatic process of maxilla, jugal bone and zygomatic process of squamosa. In Hemiechinus auritus, the jugal bone is a slender stout bone and connects the maxilla anteriorly with the squamosal posteriorly. While, it is a slightly broad bone in Cavia porcellus and it is a stout narrow curved bone in Mustela nivalis. The two pterygoids meet at the midventral line of the skull. The palatine bones enclose the vomer. The vomer bone forms the basal parts of the nasal septum $^{(9)}$. It is located in the mid-ventral line of the skull and articulates posteriorly with the presphenoid. The premaxillae and maxillae form the anterior part of the facial region. The teeth which arranged on the upper and lower jaw are heterodont ${ }^{(24)}$. The lower jaw consists of two rami. Each ramus is formed from the dentary bone which extends to articulate with the upper jaw ${ }^{(\mathbf{6})}$.There are three distinct processes associated with the mandible which are: articular process, coronoid and angular. The two rami (mandibles) are attached anteriorly by the mandibular symphysis. The mandibular symphysis is a bony structure in the three skulls under this study ${ }^{25}$. This symphysis is partially ossified in camel ${ }^{(\mathbf{1 5})}$. The Koala, Wombat and Wallaby lack the mandibular symhysis ${ }^{(26)}$.

The dental formula of the three skulls under investigation is; (I 1/1, C0/0, P1/1, M3/3) $\mathrm{x} 2=20$ teeth (Cavia porcellus), (I 3/3, C1/1, P3/2, M3/3) $\mathrm{x} 2=38$ teeth ( Hemiechinus auritus) and (I 3/3, C1/1, P3/3, M1/2)x2 = 
34 teeth(Mustela nivalis). It in Gerbils, the dental formula is $\mathrm{I} 1 / 1, \mathrm{C} 0 / 0, \mathrm{P} 0 / 0, \mathrm{M} 3 / 3) \times 2=16$ teeth $^{(27)}$.

\section{CONCLUSION}

The present study showed the special shape of each skull in the three animals under investigation. This may be due to the differences of their feeding habits. Cavia porcellus is herbivorous, Hemiechinus auritus is insectivorousand Mustela nivalis vulgaris is carnivorous. Since, the broad zygomatic arch in Cavia porcellus helps in the efficiency of the chewing of herbs. The long facial region of Hemiechinus auritus helps in catching insects. The short facial region of the Mustela nivalis and the prominent sagittal crest supports the power of pitting action during the animal's feeding. Finally, this comparative study states the main differences and similarities between the three animal's skulls with a special interest to their feeding habits.

\section{REFERENCES}

1.Yahaya A,Olopade JO and Kwari HD (2013): Morphological analysis and osteometry of the foramen magnum of the one-humped camel (Camelus dromedarius). Anat.Histol.Embryol., 42: 155-159.

2. O'Malley B(2005): Clinical Anatomy and Physiology of Exotic Species. Elsevier Saunders, USA.

3. Sarma K, Nashiruddullah $N$ and Islam $S$ (2001): Anatomy of the skull of a leopard cat (Felis bengalensis). Indian J. Ani.Sci., 71(11):1011-1013.

4. Saber AS andFernandoLB (2014): Anatomical and Radiological study on the skull of the Koala (Phascolarctos cinereus). J. Vet. Anat., 7: 1-16.

5. YiSJ, LeeHS, Kim KSet al. (1998): The comparative anatomical study of the parietal region of the skull of the Korean native goat (Capra Hircus). Anat.Histol. Embryol., 27: 323-325.

6. KumawatR, JoshiS, Mathur $R$ et al. (2014): Gross anatomical studies on the cranial bones of skull in chital (Axis axis). Indian J.Vet.Anat., 26(1):54-55.

7. IsmailS(2000): Comparative macroscopic and microscopic studies on some Egyptian vertebrates. M.Sc.Thesis.Fac.Sci.-Al-Azhar Univ., Cairo, Egypt.

8. Yousefi MH (2016): Anatomical study of the Iranian brown bear's skull (Ursus arctos): A case report. Iran J. Vet. Med., $10: 237-244$.

9. TaiboA (2019): Veterinary Medical Terminology. Guide and Workbook. $2^{\text {nd }}$ edition. John Wiley and Sons.London.
10. PandeyY, VaishR, Pandey Aet al. (2017): Gross morphometrical studies on cranial bones in tiger. Bioscan., 12:811-814.

11. Dee fails A and MageeC (2018): Anatomy and physiology of Farm Animals $8^{\text {th }}$ edition. John Wiley and Sons. London.

12. ChoudharyOP and Singh I (2016): Morphological and radiographic studies on the skull of Indian blackbuck (Antilope cervicapra). Int.. Morphol., 34:775-783.

13. Hildebrand M (1968): Anatomical Preparations. University of California Press. Berkely CA. USA.

14. Mohamed R (2018): Anatomical and radiographic study on the sSkull and mandible of the common opossum (Didelphis marsupialis(Linnaeus, 1758). Vet.Sci., 5: 44-50.

15. Singh J and Patel MR (1984): The osteology of skull of goat. Indian J. Vet. Sci. Ani Hus., 8:80-89.

16. Karan M, Timurkaan S, Özdemir D et al.(2006): Comparative macroanatomical study of the neurocranium in some carnivore. Anat. Histol. Embryol., 35:53-55.

17. Dyce KM,Sack WO and Wensing CJG (2010): Textbook of Veterinary Anatomy. $4^{\text {th }}$ edition. Elsevier Saunders. USA.

18. Madkour G (1986): Two micro-chiropterans and a carnivore from Qatar.Zoologischer Anzeiger, 216: 72-80.

19. AtalarO, UstundagY, YamanMet al. (2009): Comparative anatomy of the neuro-cranium in some wild carnivore. . Ani. Vet. Adv., 8:1542-1544.

20. HeT, FriedeH andKiliaridisS (2002): Macroscopic and roentgenographic anatomy of the skull of the ferret (Mustela putoris furo). Lab. Anim., 36:86-96.

21. Kalita PC, KalitaHC and Sarma K (2006): Anatomy of the skull of sloth bear (Melursus ursinus). Indian J. Anim.Sci., 76:225-227.

22. Koenig HE and LiebichHG (2007): Veterinary Anatomy of Domestic Mammals (Textbook and Colour Atlas). $3^{\text {rd }}$ edition, Schattauer GmbH Stuttgart. Germany.

23. Sarma K (2006): Morphological and craniometrical studies on the skull of Kagani goat (Capra hircus) of Jammu region. In.J. Morphol., 24:449-455.

24. MansourNM(2000): Anatomical cytogenic and histological studies on Egyptian mongoose, Herpestes ichneumon, (Linnaeus, 1758). M.S. Thesis.Fac. Sci., AlAzhar University (for girls), Cairo, Egypt.

25. MohamedR(2019): Anatomical and Radiographic Study on the Skull and Mandible of the African Lion (Panthera leo). J. Morphol. Scik., 36:174-181.

26. SaberAS (2015): Clinical anatomy of the mandible of three marsupial species (Koala, Wombat, Wallaby). J.Vet.Anat., 8:1-11.

27. MadkourG (1984): Chondral and osteological structures in the cranial region of common Qatarian gerbils. Zoologischer Anzeiger, 213:247-257. 